\title{
Network Pharmacology-Based Investigation of Potential Targets of Astragalus-Angelica Compound Acting on Diabetic Nephropathy
}

\section{Youzi Dong}

Shandong University of Traditional Chinese Medicine

Quanlin Zhao ( $\sim$ 2364465982@qq.com )

Affiliated Hospital of Shandong University of Traditional Chinese Medicine

\section{Research Article}

Keywords: network pharmacology, astragalus-angelica compound, diabetic nephropathy, molecular docking

Posted Date: January 11th, 2021

DOl: https://doi.org/10.21203/rs.3.rs-142572/v1

License: (c) (i) This work is licensed under a Creative Commons Attribution 4.0 International License. Read Full License 


\section{Abstract}

Through network pharmacology and molecular docking to explore the mechanism of astragalus-angelica compound in the treatment of diabetic nephropathy (DN). Screen the components and targets of astragalus and angelica compound on the TCMSP and the BATMAN-TCM, and use Cytoscape 3.7.2 to establish a component-target interaction network. Relevant targets of DN were searched through related databases, and the common targets of astragalus-angelica compound prescription and DN were obtained after comparison. The target protein interaction analysis and visualization processing were performed, and gene ontology (GO) analysis and Kyoto Encyclopedia of Gene and Genome (KEGG) pathway enrichment analysis were performed through David database, and molecular docking was performed using PyMoL and AutoDock Vina software. Through network pharmacology screening, 142 main targets of astragalusangelica compound in the treatment of DN have been identified. KEGG pathway enrichment analysis shows that the above key targets are related to apoptosis, oxidative stress, inflammation, insulin resistance and other related pathways. Molecular docking shows that the target protein has a good combination with the main active ingredients of astragalus-angelica compound. Astragalus-angelica compound may act on VEGFA, TP53, IL-6, TNF, mark1 and other targets to treat DN by regulating apoptosis, oxidative stress, inflammation, glucose and lipid metabolism and other pathways. Research methods based on network pharmacology and molecular docking provide new ideas for the pathogenesis and treatment of DN.

\section{Introduction}

Diabetic nephropathy(DN) is a common microvascular complication of diabetes and one of the important causes of end-stage renal disease (ESRD). Kidney damage caused by diabetes can affect almost all structures of the kidney, and the disease progresses much faster than non-DN patients, and the long-term prognosis is poor. Clinical treatment focuses on controlling blood sugar and blood pressure, improving renal function, and reducing urine protein excretion ${ }^{[1]}$. Our previous studies have found that the medicine for invigorating qi and promoting blood circulation can lower blood sugar and delay the progress of apoptosis caused by high glucose ${ }^{[2-3]}$. In this study, network pharmacology methods combined with molecular docking were used to establish the astragalus and angelica compound-DN-target interaction network to provide a basis for further exploration in the etiological study of DN and the interpretation of the mechanism of astragalus and angelica compound in the treatment of this disease.

\section{Methods}

\subsection{Data Preparation}

The Traditional Chinese Medicine Systems Pharmacology Database and Analysis Platform(TCMSP ${ }^{[4]}$, http://tcmspw.com/tcmsp.php) is a unique Chinese herbal medicine system pharmacology platform, which highlights the role of system pharmacology in traditional Chinese medicine. We used this database to capture the relationship between Astragalus and Angelica compound, target and DN.We retrieved all the components of the Astragalus-Angelica compound prescription from the TCMSP, and the compound's oral 
bioavailability $(O B) \geq 30 \%$ and drug-likeness $(D L) \geq 0.18$ is used as the screening condition ${ }^{[5]} \cdot A$ Bioinformatics Analysis Tool for Molecular mechANism of Traditional Chinese Medicine (BATMAN-TCM ${ }^{[6]}$, http://bionet.ncpsb.org/batman-tcm/) is the first online bioinformatics analysis tool specially designed for the research of molecular mechanism of TCM. For we-submitted astragalus-angelica compound, BATMANTCM will first predict potential targets for each ingredient, and then perform functional analyses on the above targets, astragalus and angelica ingredient-target-DN association networkwill be shown. These functions aim to contribute to the understanding of the "multi-component, multi-target and multi-pathway" combinational therapeutic mechanism of astragalus-angelica compound and to provide clues for the following experimental validation. We searched astragalus and angelica in BATMAN-TCM. The search conditions were Score cutof $\mathrm{f} \geq 20, \mathrm{P}$-value cutoff $₫ 0.05^{[7]}$, and the compound composition complies with $\mathrm{OB} \geq 30 \%$ and $\mathrm{DL} \geq 0.18$. The component prediction targets of the two databases were merged to establish the astragalus and angelica compound-component-target database, and the Cytoscape 3.7.2 software was used to construct the network diagram.

Search for DN-related targets in the Comparative Toxicogenomics Database (CTD, http://ctdbase.org/), the Online Mendelian Inheritance in Man database (OMIM, https://www.omim.org/) and the Human Gene Database (GeneCards, https://www.genecards.org/), and take the intersection with the astragalus-angelica compound targets.

\subsection{Network Construction}

Collect the common targets and get the astragalus and angelica compound-target-DN network interaction,and import the targets into STRING ${ }^{[8]}$ (https://string-db.org/), which is a database of known and predicted protein-protein interactions(PPI), and screen on the condition that the confidence score score $>0.9$. The above results were imported into Cytoscape 3.7.2 software for visualization, and the MCODE plug-in was used to screen important PPI network modules, and $\mathrm{P}<0.05$ indicated that the difference was statistically significant ${ }^{[9]}$. Use the David database (https://david.ncifcrf.gov/) and the BINGO plug-in to analyze the GO function (cell function, molecular function and biological function) and KEGG pathway enrichment analysis of the astragalus-angelica compound and the co-action target of DN. Both are statistically significant with $\mathrm{P}<0.05$.

\subsection{Molecular Docking}

Select the top 5 target proteins in the PPI network, and the core active ingredients and DN clinical recommended drugs (Irbesartan,Invokana,benazepril,captopril) searched in DRUNGBANK ${ }^{[10]}$ (https://go.drugbank.com/) for molecular docking. Download the pdb format file of the 3D structure of the target in the RCSB PDB (https://www.rcsb.org/) database, and download the mol2 format file of the 3D structure of the core active ingredient from the TCMSP database.Download the sdf format file of the 2D structure of the drugs from the DRUNGBANK database, and use Open Babel to convert the molecular structure to the mol2 format file. Using PyMoL and AutoDock Vina software ${ }^{[11]}$ to perform molecular docking with the main active ingredients, and evaluate the binding activity of the main active ingredients of astragalus-angelica compound with the target protein with the lowest binding efficiency. 


\section{Results}

\subsection{Data Preparation}

Combine the search results in TCMSP and BATMAN-TCM to obtain 208 components of astragalusangelica compound (Figure 1). Screened with $\mathrm{OB} \geq 30 \%$ and $\mathrm{DL} \geq 0.18$, and calculated the percentage of active ingredients in astragalus-angelica compound that meet the requirements and the final inclusion percentage (Figure 2). A total of 22 components were obtained (Table 1), including 2 components of angelica and 20 components of astragalus. There are 634 core prediction targets, including 93 prediction targets of angelica and 541 prediction targets for astragalus, and 58 common targets for the two (Figure $3)$.

Table 1 The top 10 components of OB value in Astragalus-Angelica Compound

\begin{tabular}{|llll|}
\hline Mol_ID & Component Name & OB (\%) & DL \\
\hline MOL000398 & isoflavanone & 109.99 & 0.3 \\
\hline MOL000378 & 7-O-methylisomucronulatol & 74.69 & 0.3 \\
\hline MOL000392 & formononetin & 69.67 & 0.21 \\
\hline MOL000433 & fa & 68.96 & 0.71 \\
\hline MOL000438 & (3R)-3-(2-hydroxy-3,4-dimethoxyphenyl)chroman-7-ol & 67.67 & 0.26 \\
\hline MOL000380 & (6aR,11aR)-9,10-dimethoxy-6a,11a-dihydro-6H-benzofurano[3,2- & 64.26 & 0.42 \\
\hline MOL000211 & mairin & 55.38 & 0.78 \\
\hline MOL000371 & 3,9-di-O-methyInissolin & 53.74 & 0.48 \\
\hline MOL000239 & jaranol & 50.83 & 0.29 \\
\hline MOL000354 & isorhamnetin & 49.6 & 0.31 \\
\hline
\end{tabular}

\subsection{Network Construction}

After further screening, 142 common potential targets of astragalus and angelica compound-DN were obtained, corresponding to 18 active ingredients (Figure 4). In the figure, the red nodes represent astragalus and angelica, the purple nodes represent components, and the blue nodes represent gene targets. The degree of freedom of a node represents the number of edges connected to the node in the network. There are a total of 14 components with more than 10 targets, including MOL000098 quercetin (122), MOL000422 kaempferol (51), MOL000354 isorhamnetin (27), MOL000358 $\beta$-sitosterol (27), MOL000449 bean sterols (23), MOL000371 3,9-dioxymethyl nisolin (21), these compounds may play an important role in the pathogenesis and treatment of DN. In addition, the number of target points of the 18 components are all $\geq 3$, indicating the complexity of the mechanism of astragalus-angelica compound in the treatment of $D N$. 
Import 142 common targets into STRING, and all can be queried, as shown in Figure 5 (the thickness of the line in the figure represents the strength of the force), and the result is imported into Cytoscape 3.7.2 software to gather the PPI network of the astragalus-angelica compound against DN.Perform cluster analysis on the PPI network, extract the targets with DC values greater than 1 times the median and 2 times the median in turn, and use the CytoHubba plug-in to screen out the top 10 drugs in the number of nodes (Figure 6).

$\mathrm{GO}$ analysis from the David database, the screening results of $\mathrm{P}<0.05$, obtained 502 biological processes (BP), 45 cell composition (CC), 96 molecular functions (MF). Sorted by the degree of significance from small to large, GO analysis results show that the BP of astragalus and angelica compound-DN-gene is significantly enriched in drug response, negative regulation of apoptosis process, positive regulation of RNA polymerase II promoter transcription, and deficiency Oxygen response, positive regulation of gene expression,inflammatory response, response to estradiol, aging, etc. MF is mainly enriched in enzyme binding, protein binding,transcription factor binding,protein isomerization activity, RNA polymerase II transcription factor activity, steroid hormone receptor, protein kinase binding, cytokine activity, etc. CC is mainly enriched in the extracellular space, extracellular zone, cytoplasm, mitochondria, fossa, nucleoplasm, extracellular matrix, plasma membrane, etc. (Table 2). Use the BINGO plug-in for analysis, and the results are shown in Figure 7.

Table 2 Biological Process of Astragalus-angelica Compound,DN and targets (top 10) 


\begin{tabular}{|c|c|c|c|}
\hline Category & Gene function & Count & P-Value \\
\hline BP & response to drug & 30 & $1.05 \times 10^{-22}$ \\
\hline BP & negative regulation of apoptotic process & 34 & $4.97 \times 10^{-22}$ \\
\hline BP & $\begin{array}{l}\text { positive regulation of transcription from RNA polymerase II } \\
\text { promoter }\end{array}$ & 42 & $1.68 \times 10^{-18}$ \\
\hline BP & positive regulation of transcription, DNA-templated & 32 & $2.31 \times 10^{-18}$ \\
\hline BP & response to hypoxia & 20 & $2.55 \times 10^{-16}$ \\
\hline BP & positive regulation of gene expression & 23 & $3.38 \times 10^{-16}$ \\
\hline BP & cellular response to hypoxia & 16 & $2.27 \times 10^{-15}$ \\
\hline BP & inflammatory response & 25 & $8.71 \times 10^{-15}$ \\
\hline BP & response to estradiol & 15 & $2.50 \times 10^{-14}$ \\
\hline BP & aging & 18 & $3.54 \times 10^{-14}$ \\
\hline MF & enzyme binding & 32 & $5.35 \times 10^{-24}$ \\
\hline MF & protein binding & 120 & $1.28 \times 10^{-16}$ \\
\hline MF & identical protein binding & 32 & $7.18 \times 10^{-14}$ \\
\hline MF & transcription factor binding & 21 & $2.29 \times 10^{-13}$ \\
\hline MF & protein heterodimerization activity & 20 & $1.01 \times 10^{-8}$ \\
\hline MF & $\begin{array}{l}\text { RNA polymerase II transcription factor activity, ligand-activated } \\
\text { sequence-specific DNA binding }\end{array}$ & 8 & $1.58 \times 10^{-8}$ \\
\hline MF & steroid hormone receptor activity & 9 & $1.86 \times 10^{-8}$ \\
\hline MF & protein homodimerization activity & 24 & $3.29 \times 10^{-8}$ \\
\hline MF & protein kinase binding & 17 & $9.26 \times 10^{-8}$ \\
\hline MF & cytokine activity & 12 & $2.45 \times 10^{-7}$ \\
\hline $\mathrm{CC}$ & extracellular space & 48 & $1.42 \times 10^{-19}$ \\
\hline $\mathrm{CC}$ & membrane raft & 16 & $5.96 \times 10^{-11}$ \\
\hline CC & extracellular region & 39 & $2.06 \times 10^{-10}$ \\
\hline $\mathrm{CC}$ & cytosol & 58 & $3.83 \times 10^{-10}$ \\
\hline
\end{tabular}




\begin{tabular}{|llcc|} 
CC & mitochondrion & 32 & $1.96 \times 10^{-8}$ \\
\hline CC & caveola & 9 & $3.46 \times 10^{-8}$ \\
\hline CC & nucleoplasm & 45 & $1.13 \times 10^{-6}$ \\
\hline CC & extracellular matrix & 13 & $3.09 \times 10^{-6}$ \\
\hline CC & plasma membrane & 56 & $6.49 \times 10^{-6}$ \\
\hline CC & nucleus & 67 & $9.72 \times 10^{-6}$ \\
\hline
\end{tabular}

According to $\mathrm{P}<0.05$ and combined with related literature, the KEGG enrichment analysis pathway was screened, and 120 pathways related to DN were obtained. Sort by the number of target genes contained in the pathway, and select pathways with a number of genes> 20 (Figure 8), including cancer pathway, PI3KAkt signaling pathway, proteoglycan in cancer, HIF-1 signaling pathway, and tumor necrosis factor signaling pathway, etc. There are 43 disease-related pathways, including type 1 diabetes, type 2 diabetes, pancreatic cancer, rheumatoid arthritis, hepatitis B, hepatitis C, non-alcoholic fatty liver, cancer pathway, tuberculosis, etc.; inflammation-related pathways such as TNF signaling pathway, nuclear transcription Factor-KB (NF-KB) signaling pathway; in addition, there are hypoxia inducible factor-1 (HIF-1) signaling pathway, insulin resistance, cyclic adenosine monophosphate (cAMP) signaling pathway, etc. Astragalusangelica compound may act on these pathways to treat DN.

\subsection{Molecular Docking}

Among the 18 main active ingredients, select the top 5 target proteins (IL-6, TP53, VEGFA, TNF, MARK1) in the PPI network and degree> 10 in the "Astragalus and Angelica compound-component-DN target interaction network". The active ingredients (quercetin, kaempferol, isorhamnetin, etc.) were molecularly docked using AutoDock Vina software, as shown in Table 3.

It is generally believed that the greater the absolute value of the docking score during molecular docking, the stronger the affinity of the compound to the target and the more stable the conformation. An absolute value greater than 4.25 indicates a certain binding activity, greater than 5.0 indicates a good binding activity, and greater than 7.0 indicates a strong binding activity ${ }^{[12]}$. The molecular docking results showed that the binding ability of quercetin, kaempferol, and isorhamnetin to TP53 was stronger, and the binding energy of each active ingredient was stronger than that of captopril. The binding energy of $\beta$-sitosterol and stigmasterol to IL- 6 is higher than that of benazepril, captopril and irbesartan. The binding ability of each active ingredient with VEGFA is low, but they are all stronger than captopril. Quercetin, formononetin, mullein, kaempferol and TNF have stronger affinity than Invokana, benazepril and captopril.

Comprehensive analysis shows that the docking scores of quercetin and TP53 and stigmasterol and IL- 6 have the largest absolute value among the five targets. The targets TP53, IL-6, VEGFA, TNF, and MARK1 respectively have the strongest affinity with quercetin, stigmasterol, kaempferol, and ivy saponins. After processing and optimization with PyMoL software, the specific binding site pattern of the target protein 
and the molecule can be seen (Figure 9). The yellow line is the hydrogen bond interaction force between the two, which is the main force that promotes the binding of the molecule to the active site.

Table 3 Docking Results of Core Target Protein and Main Active Components

\begin{tabular}{|c|c|c|c|c|c|}
\hline Compound $\backslash$ Affinity $(\mathrm{kcal} / \mathrm{mol})$ & TP53(3dcy) & $\begin{array}{l}\text { IL- } \\
6(4 \mathrm{cni})\end{array}$ & VEGFA(4kzn) & TNF(4twt) & MAPK1(6q7s) \\
\hline quercetin & -8.3 & -6.7 & -5.6 & -7.9 & -5.9 \\
\hline Jaranol & -7.6 & -6.7 & -5.3 & -7.2 & -5.3 \\
\hline hederagenin & -7.9 & -6.8 & -5.3 & -4.7 & -5.9 \\
\hline isorhamnetin & -8.1 & -6.8 & -5.5 & -7.3 & -5.8 \\
\hline beta-sitosterol & -7.2 & -8.2 & -5.2 & -5.3 & -5.4 \\
\hline 3,9-di-0-methylnissolin & -7.5 & -6.7 & -5 & -7.2 & -5.1 \\
\hline 7-0-methylisomucronulatol & -7.4 & -6.7 & -5 & -6.5 & -6 \\
\hline $\begin{array}{l}\text { (6aR,11aR)-9,10-dimethoxy- } \\
\text { 6a,11a-dihydro-6H- } \\
\text { benzofurano[3,2-c]chromen- } \\
\text { 3-ol }\end{array}$ & -8.2 & -5.9 & -4.8 & -5.6 & -5.4 \\
\hline Bifendate & -7.6 & -6.5 & -5.2 & -6.9 & -5.2 \\
\hline formononetin & -7.4 & -6.6 & -4.8 & -7.5 & -5.3 \\
\hline Calycosin & -7.4 & -6.9 & -5.1 & -7.9 & -5.4 \\
\hline kaempferol & -8.1 & -6.6 & -5.6 & -8 & -5.7 \\
\hline $\begin{array}{l}\text { 1,7-Dihydroxy-3,9-dimethoxy } \\
\text { pterocarpene }\end{array}$ & -7.4 & -7 & -5.2 & -7.5 & -6 \\
\hline Stigmasterol & -7.6 & -8.3 & -5.2 & -5.7 & -5.7 \\
\hline Benazepril & -8.4 & -6.8 & -6 & -7.2 & -6.2 \\
\hline Irbesartan & -9.3 & -7.9 & -5.7 & -9.1 & -7 \\
\hline Captopril & -6 & -4.3 & -3.5 & -5.7 & -3.8 \\
\hline Invokana & -9 & -8.4 & -6.3 & -7.4 & -6.7 \\
\hline
\end{tabular}

\section{Discussion}

Astragalus and Angelica compound prescription has rich compatibility theory and wide clinical application, and it is one of the representative traditional Chinese medicine pairs. Because it can not only form a party on its own, but also can be used as a basic drug pairing prescription, it has been valued by the doctors of the past generations. The compatibility principle of astragalus and angelica compound prescription is the 
compatibility of qi and blood, which has three functions: nourishing qi and blood, replenishing qi and invigorating blood, and replenishing qi and promoting blood circulation. Astragalus polysaccharides can

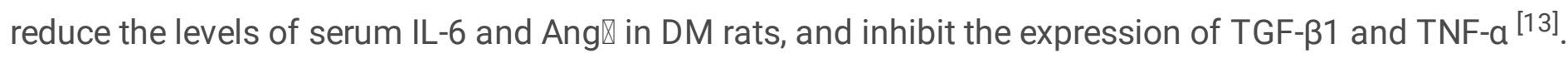
Our previous research found ${ }^{[2-3]}$ that Astragalus and Angelica compound can improve the related clinical symptoms of DN rats by regulating IL-6 and NF-KB related genes. IL-6 is a promoting factor for pancreatic islet function under normal conditions, and it is also an injury factor under pathological conditions. HIF can activate NF-KB ${ }^{[15]}$. Hypoxia can inhibit the activity of PHD1, leading to the activation of IKK, which in turn phosphorylates $\mathrm{IKB}$, and the dissociated NF-KB leads to the transcriptional activation of downstream genes, such as inflammatory factors ${ }^{[16]}$.

Through the analysis of the astragalus and angelica compound-DN-gene network analysis, we found that 31 pathways contain IL-6, of which the HIF-1 signaling pathway and insulin resistance pathway play a major role. There are 104 target genes and IL-6 The closest connection. There are 33 pathways containing TP53, of which the MAPK signaling pathway and P53 pathways play the main role, and 100 target genes are most closely related to TP53; 13 pathways contain VEGFA, of which PI3K-Akt signal pathway plays the main role, 100 target genes are most closely related to VEGFA. There are 41 pathways that contain TNF, of which the hepatitis B signaling pathway and cancer proteoglycan pathways play a major role, and 99 target genes are most closely related to TNF. Quercetin mainly acts through IL-6, VEGFA, and TP53, and the compound that acts mainly through TNF is kaempferol. Astragalus-angelica compound may act on IL-6, TP53, VEGFA, TNF and other targets to regulate TNF signaling pathway, insulin resistance, tumor necrosis factor signaling pathway, etc., by improving oxidative stress, inhibiting inflammation, and improving insulin resistance to treat glomerular disease and renal fibrosis.

\section{Conclusion}

Through analysis of the main biological active components of astragalus-angelica compound and their pharmacological mechanism, it is found that astragalus-angelica compound acts on multiple targets and proteins in signal pathways such as inflammation and oxidative stress through multiple chemical components, which can delay progression of DN. In the follow-up study, we plan to design animal pharmacological experiments in vivo and in vitro to conduct in-depth discussions on the mechanism of action of astragalus-angelica compound in the treatment of $\mathrm{DN}$, in order to provide more references for its clinical application and development.

\section{Conflicts of Interest}

We declare no competing interests.

\section{Declarations}

\section{Acknowledgments}

This work is supported by the National Natural Science Foundation of China (Nos.81774254). 


\section{References}

1. Umanath K, Lewis JB. Update on Diabetic Nephropathy: Core Curriculum 2018. Am J Kidney Dis. 2018;71(6):884-895.

2. Yin Y, Qi F, Song Z, Zhang B, Teng J. Ferulic acid combined with astragaloside IV protects against vascular endothelial dysfunction in diabetic rats. Biosci Trends. 2014;8(4):217-226.

3. Yin $\mathrm{Y}, \mathrm{Li} \mathrm{H}, \mathrm{Chen} \mathrm{Y}$, et al. Combination of Astragalus membranaceous and Angelica sinensis Ameliorates Vascular Endothelial Cell Dysfunction by Inhibiting Oxidative Stress. Evid Based Complement Alternat Med. 2020;2020:6031782. Published 2020 Sep 18.

4. Jinlong R; Peng L; Jinan W, et al. TCMSP: a database of systems pharmacology for drug discovery from herbal medicines. J Cheminformatics. 2014 Apr 16;6(1):13.

5. J. Ru, L. Peng, J. Wang, Z. Wei, B. Li, H. Chao, P. Li, Z. Guo, W. Tao, Y. Yang, TCMSP: a database of systems pharmacology for drug discovery from herbal medicines, J. Cheminform. 6 (1) (2014) 13.

6. Liu Z, Guo F, Wang Y,et al.BATMAN-TCM: a Bioinformatics Analysis Tool for Molecular mechANism of Traditional Chinese Medicine. Sci Rep. 2016, 6:21146.

7. Cai L, Kang YJ. Cell death and diabetic cardiomyopathy. Cardiovasc Toxicol[J]. 2003;3(3):219-28.

8. Szklarczyk D, Gable AL, Lyon D, et al.STRING v11: protein-protein association networks with increased coverage, supporting functional discovery in genome-wide experimental datasets. Nucleic Acids Res. 2019 Jan; 47:D607-613.

9. Z. Song, Y. Fang, B. Xiang, B. Lan, S. Cheng, Systems pharmacological approach to investigate the mechanism of Acori Tatarinowii Rhizomafor Alzheimer's Disease, Evid.-Based Complement. Alternat. Med. 2018 (4) (2018) 1-20.

10. Wishart DS, Feunang YD, Guo AC,et al.DrugBank 5.0: a major update to the DrugBank database for 2018. Nucleic Acids Res. 2017 Nov 8.

11. Trott $0 \otimes O$ Ison AJ. AutoDock Vina: improving the speed and accuracy of docking with a new scoring

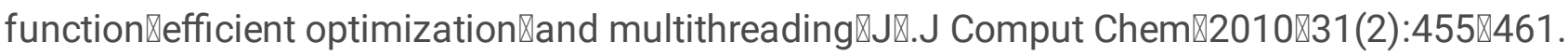

12. Hsin $\mathrm{K}$ Y, Ghosh $\mathrm{S}$, Kitano $\mathrm{H}$. Combining machine learning systems and multiple docking simulation packages to improve docking prediction reliability for network pharmacology [J].PLoS One,2013,8(12):e83922.

13. Chen W, Li Y-, Yu M-. Astragalus polysaccharides: an effective treatment for diabetes prevention in NOD mice. Exp Clin Endocrinol Diabetes. 2008 Aug;116(8):468-74.

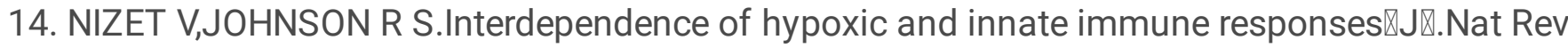
Immunol,2009,9(9):609-617ه

15. Cummins EP,Berra E,Comerford KM,et al. Prolyl hydroxylase-1 negatively regulates IkappaB kinase-

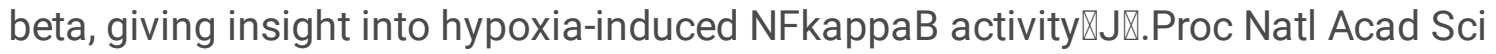
USA.2006;103(48):18154-18159.

\section{Figures}




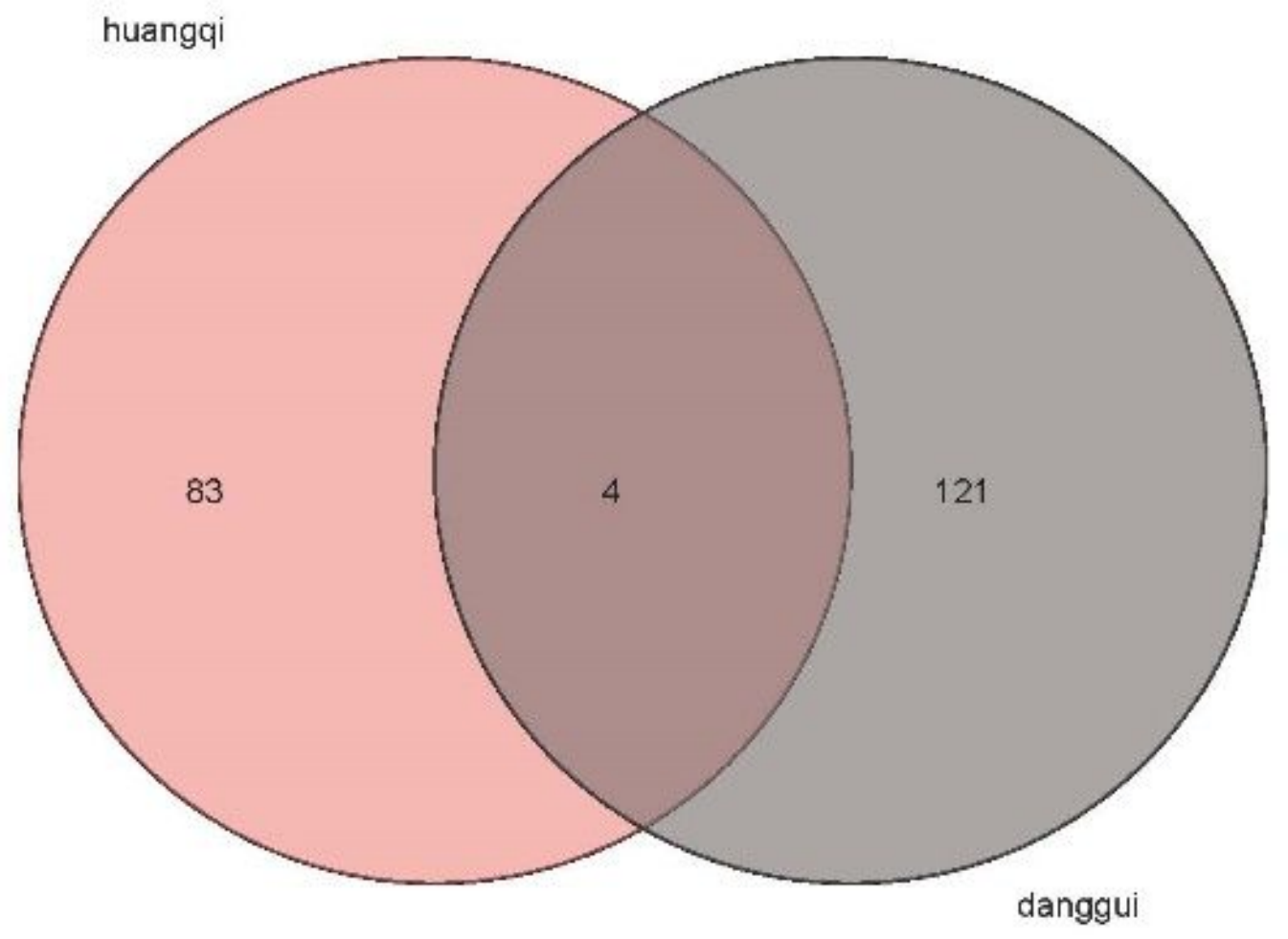

Figure 1

Venn Diagram of Compound Screening

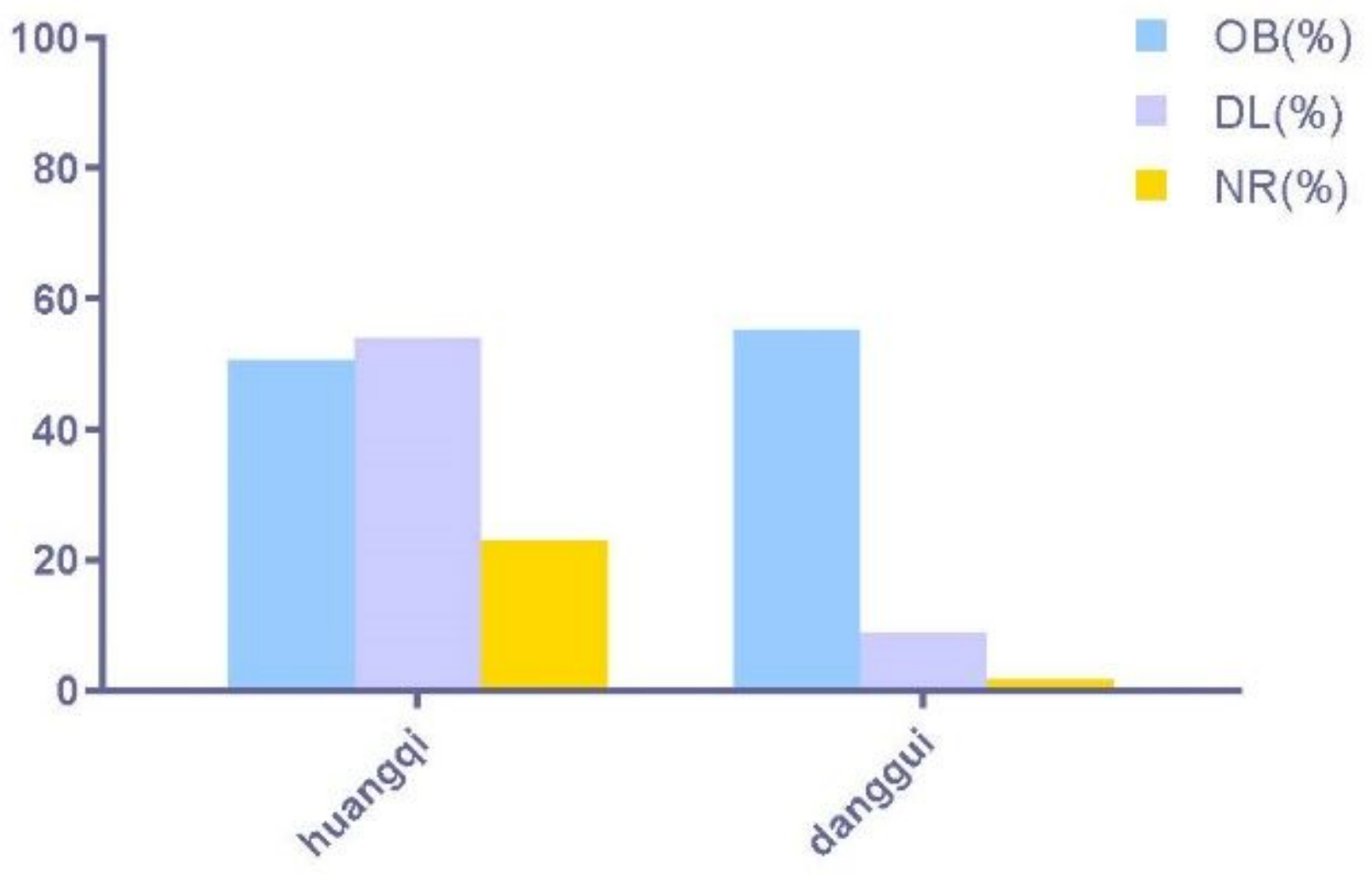

Figure 2 


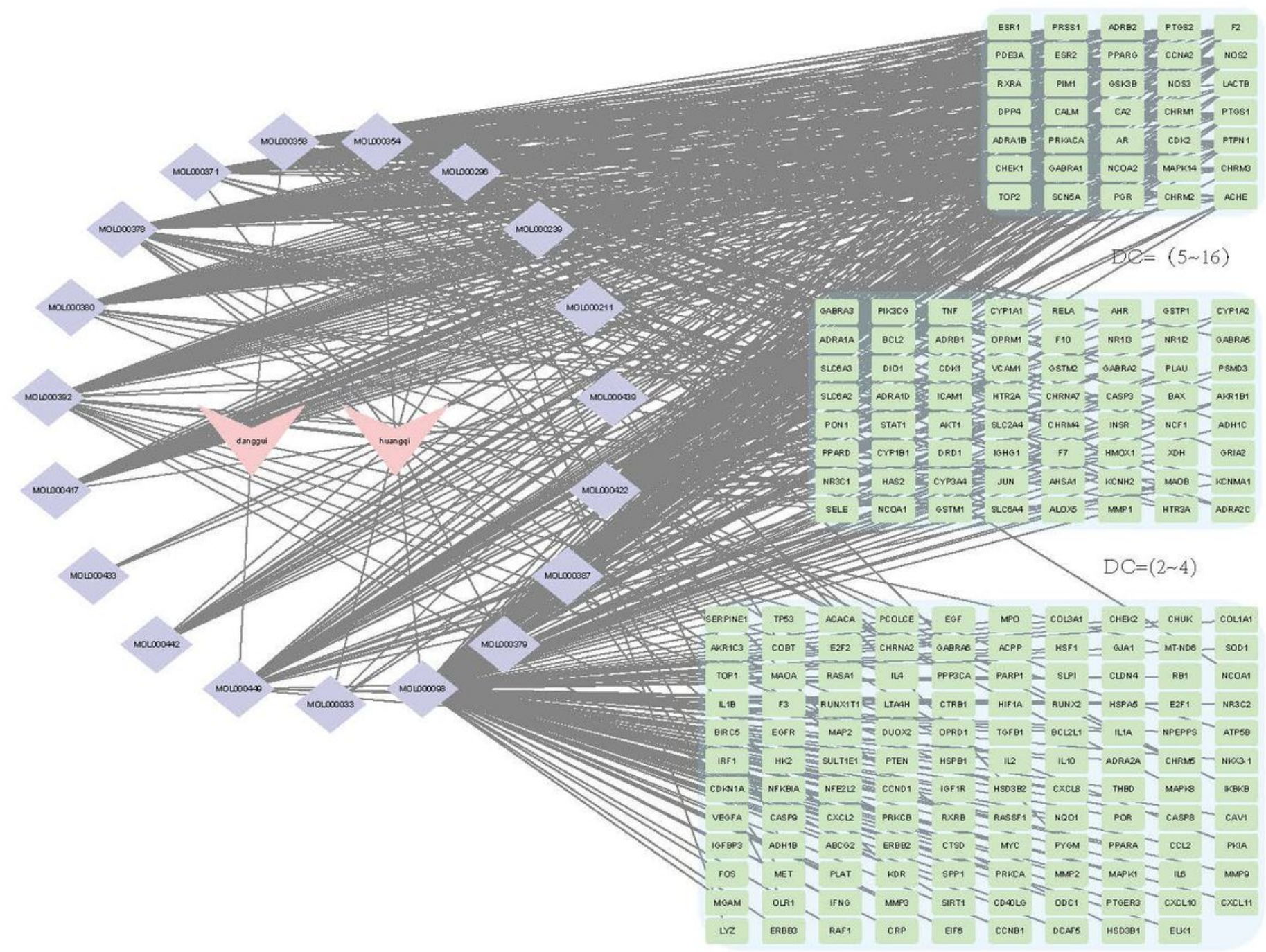

$\mathrm{DC}=1$

Figure 3

Interaction Network of Astragalus\&Angelica Compound-Components-Targets Searched in TTD, DrugBank, DisGeNET, CTD, OMIM and GeneCards databases, a total of 1993 human targets related to DN were obtained. 


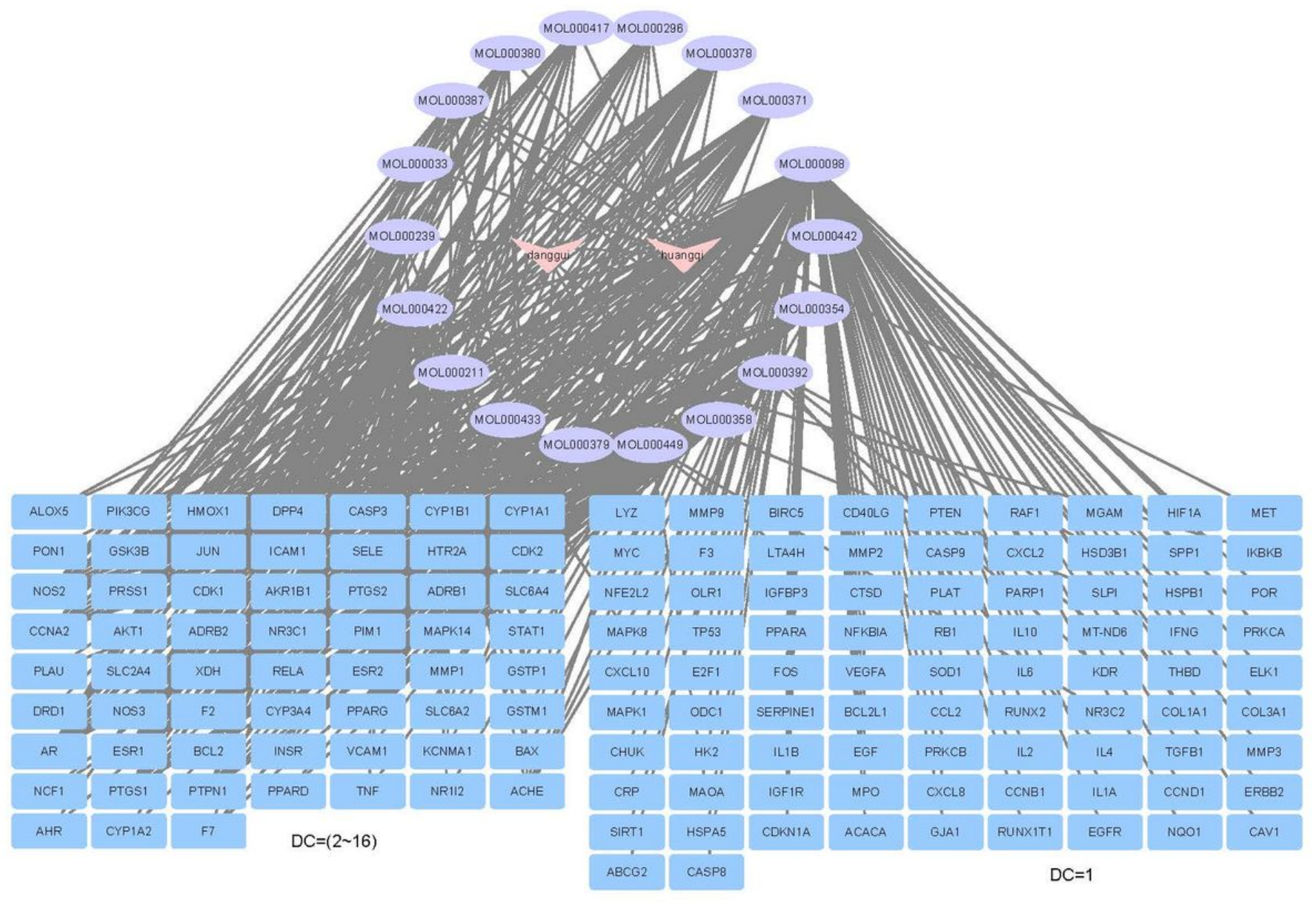

\section{Figure 4}

Interaction Network of Astragalus\&Angelica Compound-Components-DN Targets 


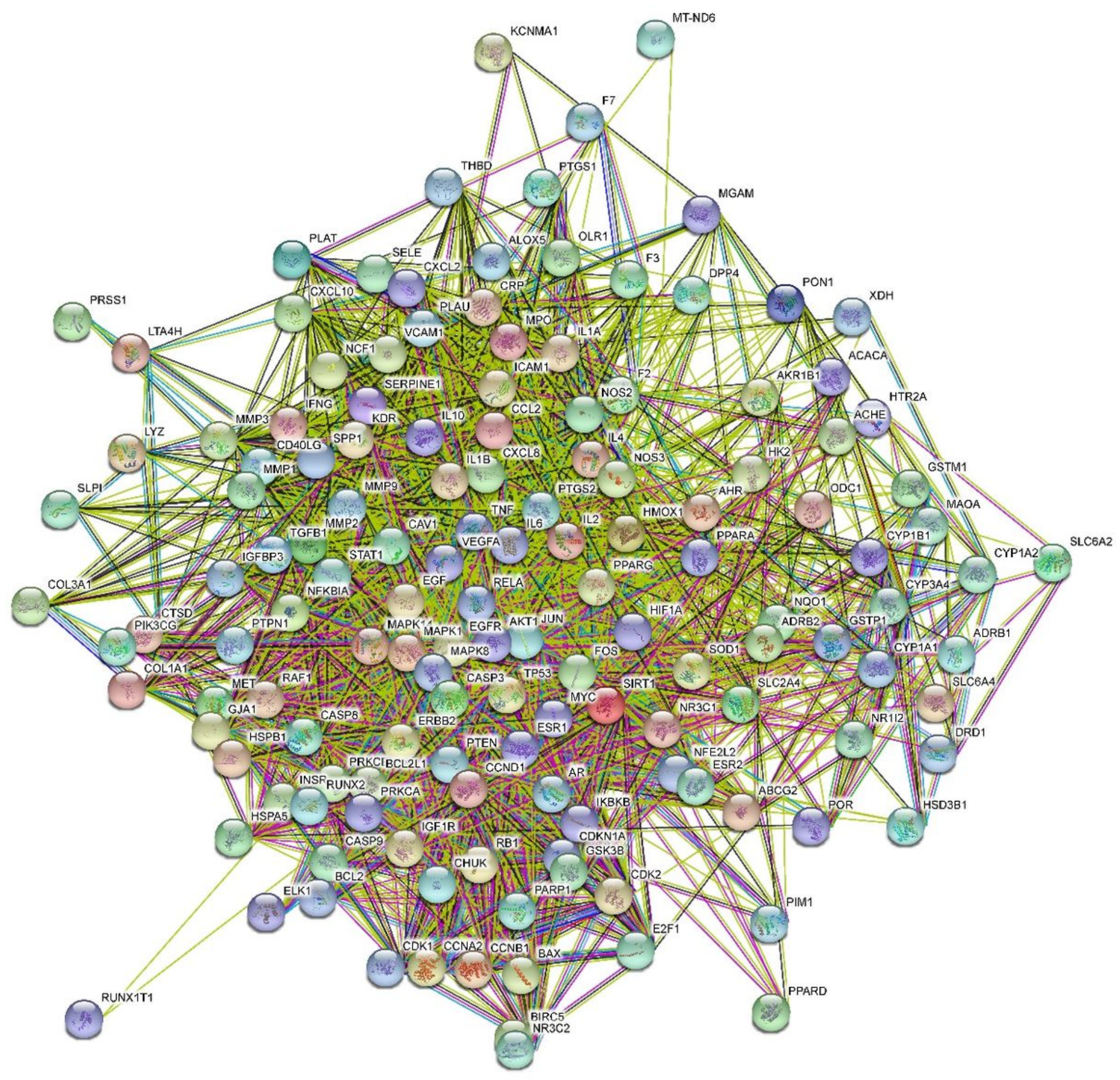

Figure 5

PPI Network 


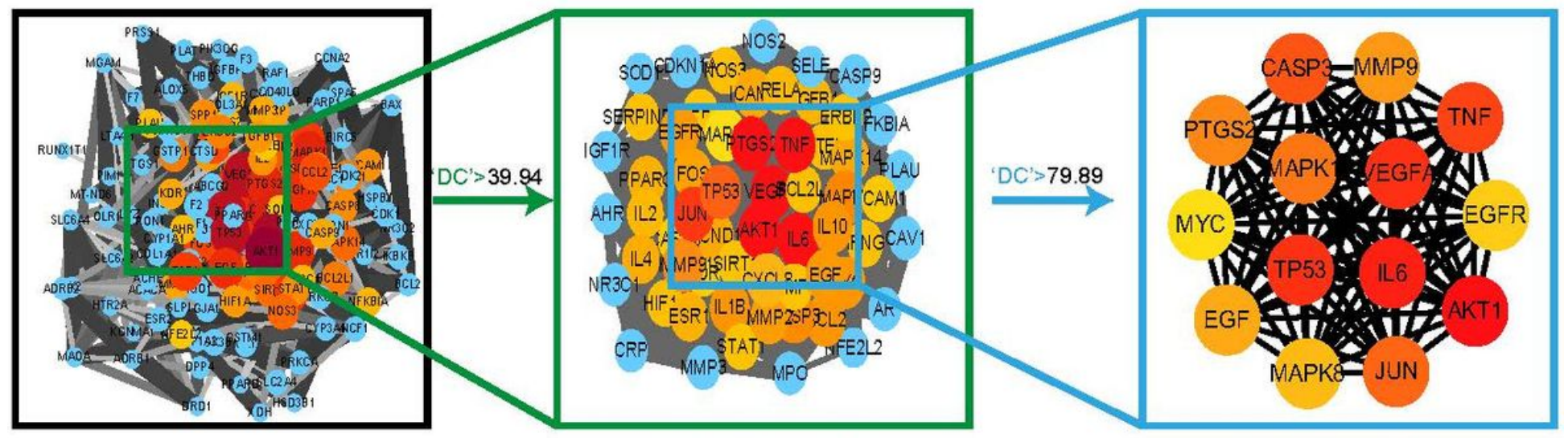

142 nodes and 2836 edges

60 nodes and 1361 edges

14 nodes and 91 edges

Figure 6

Core Target Topology

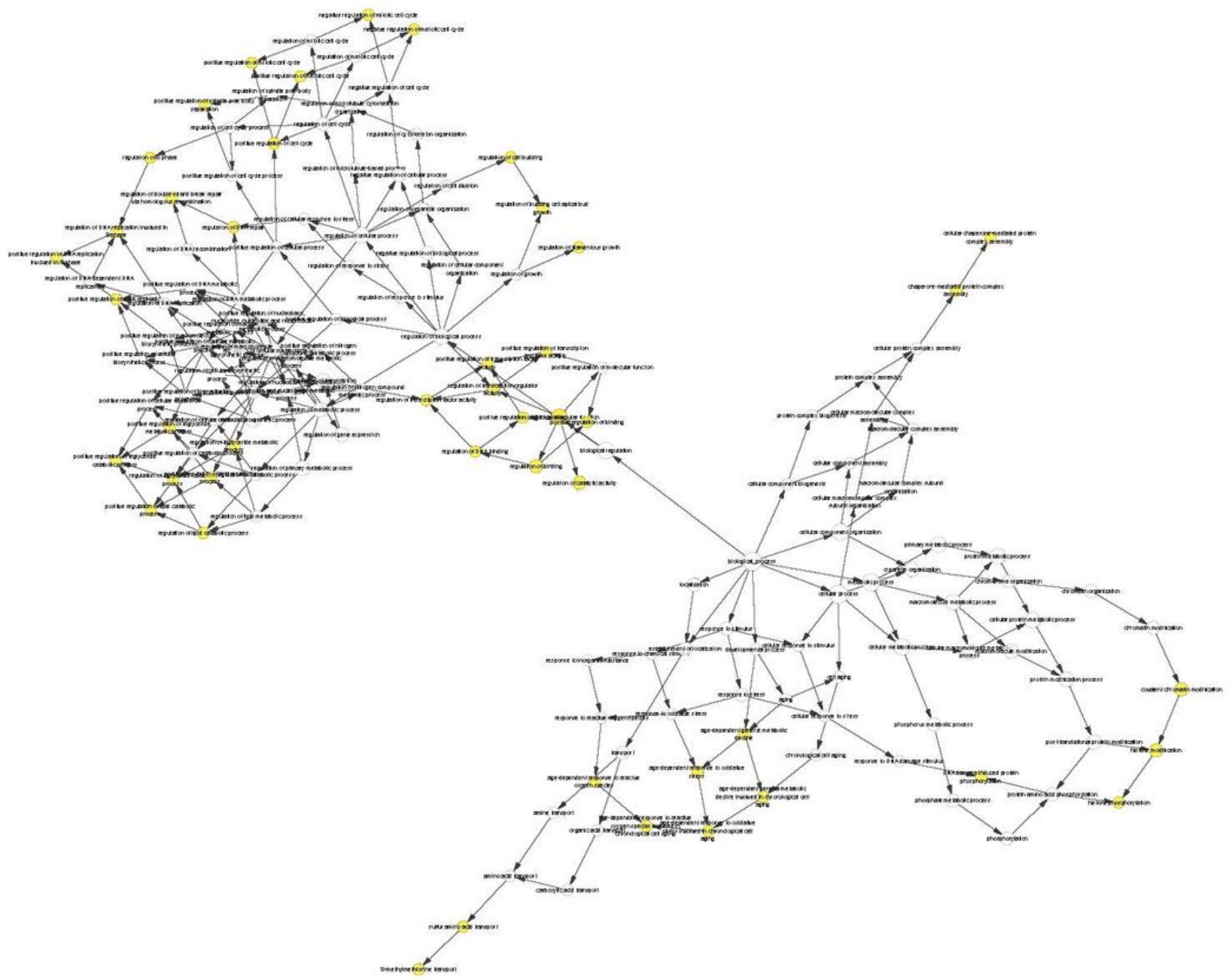

Figure 7

Functional Enrichment Analysis 


\section{Top 17 of KEGG Enrichment}

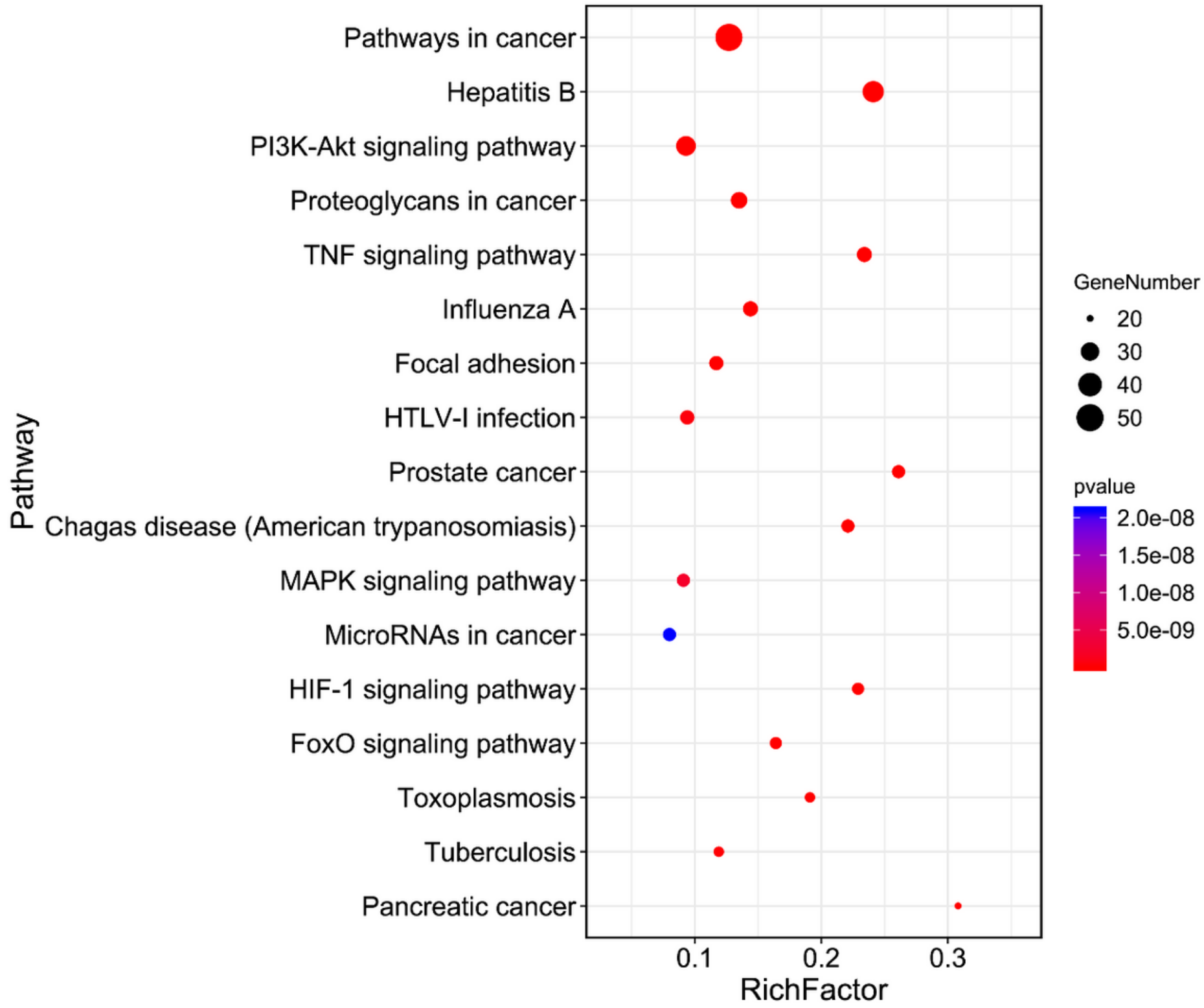

Figure 8

Biological Pathway of Astragalus-angelica Compound,DN and targets (count $>20$ ) 

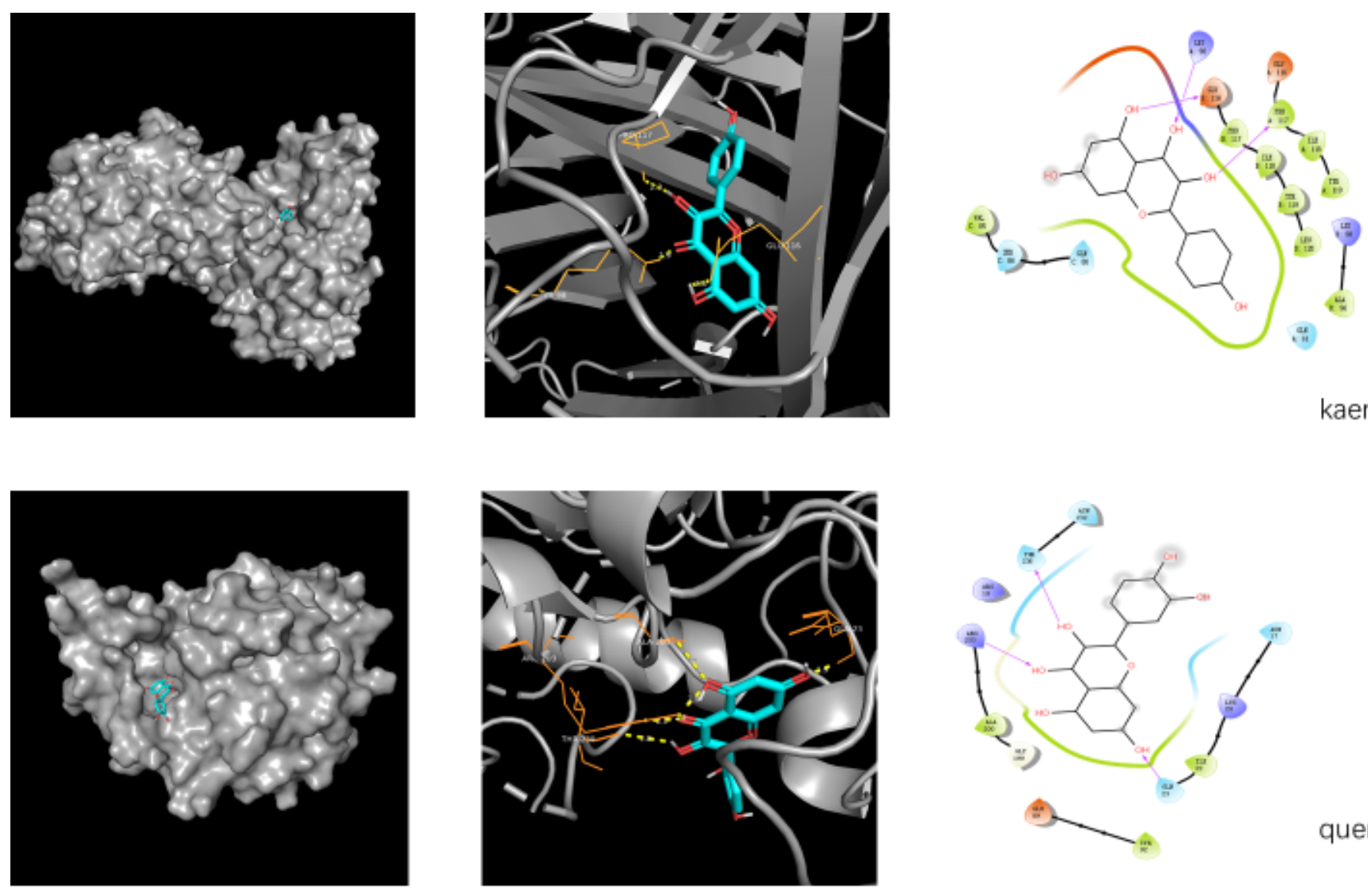

kaempferol -TNF
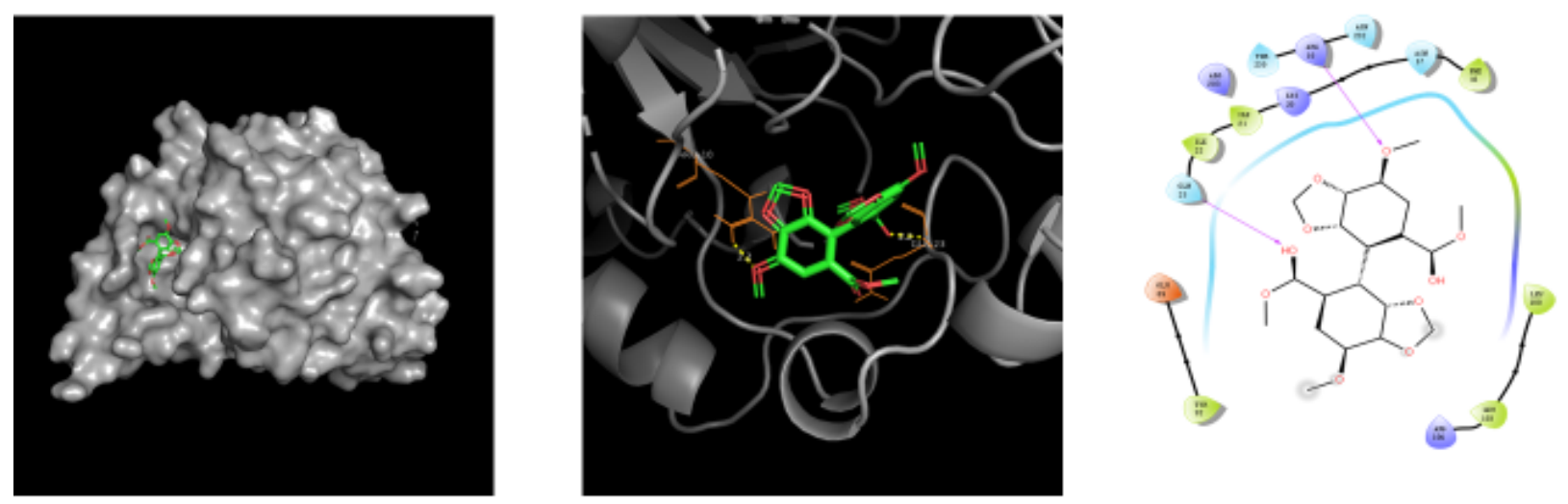

quercetin -TP53

bifendate -TP53

Figure 9

Molecular Docking Patterns 